\title{
Fibrinolysis in patients with a mild-to-moderate bleeding tendency of unknown cause
}

\author{
Johanna Gebhart ${ }^{1}$ - Sylvia Kepa ${ }^{1}$ - Stefanie Hofer ${ }^{1} \cdot$ Silvia Koder $^{1}$ • Alexandra Kaider ${ }^{2}$. \\ Alisa S. Wolberg ${ }^{3} \cdot$ Helmuth Haslacher ${ }^{4}$ Peter Quehenberger ${ }^{4} \cdot$ Ernst Eigenbauer $^{5}$. \\ Simon Panzer ${ }^{6} \cdot$ Christine Mannhalter $^{7} \cdot$ Ingrid Pabinger $^{1}$
}

Received: 18 October 2016 / Accepted: 29 November 2016 /Published online: 26 December 2016

(C) The Author(s) 2016. This article is published with open access at Springerlink.com

\begin{abstract}
In more than $50 \%$ of patients with a mild-tomoderate bleeding tendency, no underlying cause can be identified (bleeding of unknown cause, BUC). Data on parameters of fibrinolysis in BUC are scarce in the literature and reveal discrepant results. It was the aim of this study to investigate increased fibrinolysis as a possible mechanism of BUC. We included 270 patients ( 227 females, median age 44 years, 25 $75^{\text {th }}$ percentile $\left.32-58\right)$ with BUC and 98 healthy controls $(65$ females, median age 47 years, $25-75^{\text {th }}$ percentile $\left.39-55\right)$. Tissue plasminogen activator (tPA-) antigen and activity, plasminogen activator inhibitor type-1 (PAI-1), tPA-PAI-1 complexes, thrombin activatable fibrinolysis inhibitor (TAFI), $\alpha 2$ antiplasmin, and D-dimer were determined. While PAI-1 deficiency was equally frequent in patients with BUC and
\end{abstract}

Ingrid Pabinger

ingrid.pabinger@meduniwien.ac.at

1 Department of Medicine I, Clinical Division of Hematology and Hemostaseology, Medical University Vienna, Waehringer Guertel 18-20, 1090 Vienna, Austria

2 Center for Medical Statistics, Informatics and Intelligent Systems, Medical University Vienna, Vienna, Austria

3 Department of Pathology and Laboratory Medicine, University of North Carolina, Chapel Hill, NC 27599-7525, USA

4 Department of Laboratory Medicine, Division of Medical and Chemical Laboratory Diagnostics, Medical University Vienna, Vienna, Austria

5 IT-Systems and Communications, Medical University Vienna, Vienna, Austria

6 Department of Blood Group Serology and Transfusion Medicine, Medical University Vienna, Vienna, Austria

7 Department of Laboratory Medicine, Division of Molecular Biology, Medical University Vienna, Vienna, Austria controls $(91 / 270,34 \%$, and 33/98, 34\%, $p=0.996)$, tPA activity levels were more often above the detection limit in patients than in controls $(103 / 213,48 \%$, and $23 / 98,23 \%$, $p<0.0001)$. We found lower levels of tPA-PAI-1 complexes (6.86 (3.99-10.00) and $9.11(7.17-13.12), p<0.001)$ and higher activity of TAFI (18.61 (15.80-22.58) and 17.03 (14.02-20.02), $p<0.001)$ and $\alpha 2$-antiplasmin (102 (94$109)$ and $98(90-106], p=0.003)$ in patients compared to controls. Detectable tPA activity (OR 3.02, 95\%CI 1.75$5.23, p<0.0001$ ), higher levels of TAFI (OR 2.57, 95\%CI $1.48-4.46, p=0.0008)$ and $\alpha 2$-antiplasmin (OR 1.03, 95\% CI $1.01-1.05, p=0.011$ ), and lower levels of tPA-PAI-1 complexes (OR 0.90, 95\%CI 0.86-0.95, $p<0.0001$ ) were independently associated with BUC in sex-adjusted logistic regression analyses. We conclude that the fibrinolytic system can play an etiological role for bleeding in patients with BUC.

Keywords Bleeding of unknown cause $\cdot$ Hyperfibrinolysis · Plasminogen activator inhibitor-1 deficiency · Tissue plasminogen activator $\cdot$ Thrombin activatable fibrinolysis inhibitor · Alpha2-antiplasmin

\section{Introduction}

In the majority of adult patients with a mild-to-moderate bleeding tendency, no diagnosis can be established and the cause for the bleeding symptoms remains elusive [1]. This lack of a diagnosis subsequently hinders targeted therapy and causes high psychological strain in affected individuals and treating physicians, especially in situations with increased bleeding risk, such as surgery.

Fibrinolysis is a strictly regulated process modulated by activators and inhibitors. Hyperfibrinolytic conditions associated with bleeding tendencies of variable severity have been 
found in hereditary deficiencies of anti-fibrinolytic factors, such as $\alpha 2$-antiplasmin deficiency [2,3] and deficiency of the plasminogen activator inhibitor type-1 (PAI-1) [4-8]. Although hyperfibrinolysis has been discussed as a possible cause for mild-to-moderate bleeding tendencies of unknown cause, the assessment of fibrinolysis is not included in the routine coagulation work-up of patients with mild-tomoderate bleeding tendencies.

Parameters of fibrinolysis have been inconsistently investigated in few cohorts with a bleeding tendency and revealed conflicting results [9-13]. Even the analysis of the same parameter in similar cohorts (e.g. patients with menorrhagia) revealed discrepancies $[13,14]$.

Our study aimed to investigate fibrinolysis in a cohort of patients with a mild-to-moderate bleeding tendency in whom no diagnosis could be established despite thorough investigation of hemostatic parameters. We assessed a panel of factors involved in the fibrinolytic process to determine the contribution of fibrinolysis to bleeding tendencies of unknown cause (BUC). Results in patients were compared to those of healthy controls.

\section{Patients and methods}

\section{Patients}

All patients had symptoms of a mild-to-moderate bleeding tendency that were recorded within the standardized questionnaire [15], encompassing skin manifestations such as hematoma or easy bruising, increased postsurgical bleeding, prolonged bleeding after tooth extraction, small wound bleeding, epistaxis, oral mucosal bleeding, gastrointestinal bleeding (not caused by pathologies in the GI-tract), spontaneous muscle or joint bleeding, and heavy menstrual bleeding and increased postpartum bleeding in females. For the current analysis, we selected patients with a mild-to-moderate bleeding tendency in whom no diagnosis could be established from two different bleeding cohorts: the Vienna Bleeding Study (VIBS) and the Vienna Bleeding Biobank (VIBB). Both studies were conducted at the outpatient department of the Division of Hematology and Hemostaseology, Medical University Vienna, Austria and were approved by the Ethics Committee of the Medical University Vienna. All procedures were in accordance with the ethical standards of the committee on human experimentation (institutional and national) and with the Helsinki Declaration of 1975, as revised in 2008. All patients and controls gave their written informed consent.

For VIBS, patient recruitment started in November 2006 and was ongoing until March 2008. Patients who had been investigated at our center in the years 2000-2004 because of a mild-to-moderate bleeding tendency and in whom no diagnosis that would explain the bleeding tendency was established were asked to participate in the study $[16,17]$. The second cohort (VIBB) started in 2009 and included all patients with a mild-to-moderate bleeding tendency who were referred to the outpatient department for further investigations. A cohort of 98 unrelated subjects without a history of bleeding and/or thromboembolic events was recruited from hospital personnel, their friends, and relatives.

Patients and controls underwent a structured interview on their medical history in general and their personal and familial bleeding history in particular upon study inclusion.

Exclusion criteria that applied for patients and controls were antithrombotic and/or anti-inflammatory therapy, impaired liver function (prothrombin time $<75 \%$ of normal due to a decrease in vitamin $\mathrm{K}$ dependent clotting factors), severe renal insufficiency, and/or thrombocytopenia $\left(<100 \times 10^{9} / \mathrm{L}\right)$. Pregnant women, subjects with active malignancy, those with surgery or delivery within 6 weeks, bacterial infection within 2 weeks prior to recruitment, or acute phase reaction at recruitment were not included. Gastrointestinal (GI) bleeds due to pathologies in the GI tract or intracerebral bleeding due to aneurysm were not regarded as spontaneous bleeding.

\section{Selection of patients with bleeding of unknown cause (BUC)}

Patients with vWF antigen and/or activity levels of $\leq 50 \%$; pathologies in the assessment of platelet function by light transmission aggregometry and/or glycoprotein expression by flow cytometry; deficiencies of the clotting factors VIII, IX, or XIII $\leq 50 \%$; or the diagnosis of any other known inherited or acquired coagulation disorder were not included in this analysis. In the VIBS, as previously described, 135 patients were screened for eligibility [17]. Of them, 101 patients fulfilled the inclusion criteria and were enrolled in the study. Finally, 74 patients (73\%), in whom no diagnosis according to the criteria could be established and the assessed parameters of fibrinolysis were available, were included in this data analysis. Until November 2013, 277 patients were included in the VIBB. No bleeding disorder could be found in 196 patients (71\%). Thus, 270 patients with BUC were identified in the two-patient cohorts with mild-to-moderate bleeding tendencies. In the present case-control study, results were compared to those of 98 unrelated healthy controls.

\section{Evaluation of the bleeding severity in patients with BUC}

A standardized questionnaire, previously described [15], was completed by a trained physician within a structured patient interview. The recorded bleeding symptoms were epistaxis, hematoma/easy bruising, small wound bleeding, oral mucosal and/or gingival bleeding, GI bleeding, postpartum bleeding, muscle and/or joint bleeding, bleeding after tooth extraction, postsurgical bleeding, and menorrhagia. All bleeding 
symptoms were scored according to their most severe occurrence. The applied bleeding score quantifies the individual bleeding tendency on a scale from 0 to 30 points.

\section{Sampling}

Blood samples were drawn with a 21-gauge butterfly needle (Greiner Bio-One, Kremsmuenster, Austria) by antecubital venipuncture into a Vacuette tube (Greiner Bio-One, Kremsmuenster, Austria) containing trisodium citrate (nine parts of whole blood, one part of trisodium citrate 3.8\%). The tubes were transferred to the MedUni Wien Biobank, a centralized sample handling and storage facility (www.biobank.at). Hence, all further processing steps occurred according to the standard operating procedures in an ISO 9001:2008 certified environment. In detail, platelet poor plasma was prepared by centrifugation at $2000 \mathrm{~g}$ for $15 \mathrm{~min}$ at $15{ }^{\circ} \mathrm{C}$ (Hettich Rotanta 460 Robotic, Tuttlingen, Germany) and as a second step at $18,000 \mathrm{~g}$ for $2 \mathrm{~min}$ (Eppendorf 5417R, Hamburg, Germany) and stored at $<-70{ }^{\circ} \mathrm{C}$.

\section{Assessment of hyperfibrinolysis}

The following parameters were investigated in patients and controls: tPA antigen $(\mathrm{U} / \mathrm{mL})$ and activity $(\mathrm{U} / \mathrm{mL})$, PAI-1 antigen $(\mathrm{U} / \mathrm{mL})$, tPA-PAI-1 complexes $(\mathrm{ng} / \mathrm{mL})$, TAFI $(\mathrm{ng} / \mathrm{mL})$, $\alpha 2$-antiplasmin (\%), fibrinogen Clauss (mg/dL), and D-dimer $(\mu \mathrm{g} / \mathrm{mL})$.

Tissue plasminogen activator (tPA) antigen and activity were assessed using a sandwich ELISA (tPA Actibind ELISA, Technoclone, Vienna) in which tPA is bound with a catching antibody that is not interfering with its functional activity. Bound tPA antigen is quantified with a peroxidaselabeled monoclonal antibody (mAB). tPA activity was determined using plasminogen, cyanogen bromide fragments of fibrinogen, and a plasmin substrate.

The active PAI-1 antigen $(\mathrm{U} / \mathrm{mL})$ was measured using a sandwich ELISA (PAI-1 Actibind ELISA, Technoclone, Vienna, Austria).

tPA-PAI-1 complexes were determined by ELISA (tPAPAI-1 Complex ELISA Kit, Technoclone, Vienna, Austria) based on catching the complexes with a $\mathrm{mAB}$ directed against tPA. For quantification, a peroxidase-labeled $\mathrm{mAB}$ against PAI-1 was used.

The measurement of TAFI was based on a chromogenic assay (Stachrom TAFI, Asnières sur Seine, France) in which TAFI is first activated to TAFIa by a human-thrombin-rabbitthrombomodulin complex and then quantified after two hydrolysation steps by measurement of discoloration at $405 \mathrm{~nm}$.

The activity of $\alpha 2$-antiplasmin was determined by the chromogenic STA Stachrom antiplasmin assay (Diagnostic Stago, Asnieres, France). Measurement was performed on a
STA-R evolution (Diagnostic Stago, Asnieres, France) at the Clinical Department of Laboratory Medicine.

Fibrinogen was quantitatively measured by the Claussmethod at the Clinical Department of Laboratory Medicine using an automated assay (STA Fibrinogen, Roche Diagnostics and Diagnostica Stago, Asnières sur Seine, France). This functional assay is based on clotting of citrated plasma in presence of an excess of thrombin, which is inversely proportional to the fibrinogen concentration. The reagent contains a heparin inhibitor. Tests were performed on the STA-R (Diagnostica Stago, Asnières sur Seine, France).

The fibrinogen degradation product D-dimer was determined by an ELISA (Asserachrom D-Di, Diagnostica Stago, Asnières sur Seine, France). Binding to a mouse-mAB is used to immobilize D-dimer. A peroxidase-labeled rabbit $\mathrm{mAB}$ against human fragment $\mathrm{D}$ was used for quantification.

\section{Statistics}

All variables representing fibrinolysis parameters are described by their median (quartile) values. To achieve normal distribution in case of right-skewed variables, log2-transformed values were used for all statistical analyses. Group comparisons with respect to the continuous variables were performed using the two-sample $t$ test. The variable tPA activity was compared using the nonparametric Wilcoxon rank sum test. The Wilcoxon rank sum test was also used to compare the assessed parameters of fibrinolysis between patients with or without specific bleeding symptoms. No correction for multiplicity was applied due to the exploratory character of this study.

Due to the high proportion of tPA activity values close to zero and below the sensitivity of the assay, tPA activity was considered as a binary variable $(0 \mathrm{U} / \mathrm{mL}$ vs $>0 \mathrm{U} / \mathrm{mL})$ within the regression models. Univariate logistic regression models as well as sex-adjusted logistic regression models were performed to evaluate the strength of the fibrinolysis variables to discriminate between patients and controls. Results of the logistic regression models are given by the estimated odds ratios (OR) with $95 \%$ confidence intervals (CI) and $p$ values. $P$ values $<0.05$ were considered statistically significant.

\section{Results}

\section{Characteristics of patients and controls}

Clinical and laboratory characteristics of patients with BUC and controls are shown in Table 1. There was a female predominance in our group of patients with BUC. Blood groups were available for 245 patients (90.7\%) and 97 healthy controls (99\%). Blood group $\mathrm{O}$ occurred with an equal frequency in patients $(42.4 \%)$ and controls $(44.3 \%)$. There was also no difference in age or body mass index (BMI) between the groups. 
Table 1 Clinical and laboratory characteristics of patients and controls

\begin{tabular}{lll}
\hline & Patients $(n=270)$ & Controls $(n=98)$ \\
\hline Female, n $(\%)^{*}$ & $227(84.1)$ & $65(66.3)$ \\
BG 0, n $(\%)^{\mathrm{a}}$ & $104(42.4)$ & $43(44.3)$ \\
Family history, n $(\%)^{\mathrm{b}}$ & $75(34.7)$ & \\
& Median $(25-75$ percentile $)$ & Median $(25-75$ percentile) \\
Age $($ years $)$ & $44(32-58)$ & $47(39-55)$ \\
BMI $\left(\mathrm{kg} / \mathrm{m}^{2}\right)$ & $23.7(21.3-27.1)$ & $23.8(21.5-25.9)$ \\
Hemoglobin $(\mathrm{g} / \mathrm{dL})^{*}$ & $13.7(12.8-14.5)$ & $13.9(13.2-14.9)$ \\
Platelet count $\left(10^{9} / \mathrm{L}\right)$ & $244(216-278)$ & $248(220-286)$ \\
PT $(\%)$ & $116(106-126)$ & $114(103-125)$ \\
APTT $(\mathrm{sec})$ & $35.2(33.2-38.0)$ & $35.5(33.3-37.4)$ \\
Bleeding score & $4(3-6)$ & - \\
\hline
\end{tabular}

$B G$ blood group, $B M I$ body mass index, $P T$ prothrombin time, $A P T T$ activated partial thromboplastin time

* Statistically significant difference between the groups

${ }^{\text {a }}$ Available of 245 patients (90.7\%) and 97 healthy controls (99.0\%)

${ }^{\mathrm{b}}$ Family history of bleeding available of 216 patients $(80.0 \%)$
Interestingly, patients had lower levels of hemoglobin. As this difference could be due to the female predominance in the patient group, we adjusted for sex, and indeed, then the difference disappeared $(p=0.71)$ (data not shown). There was no difference in platelet count or global clotting tests such as prothrombin time (PT, \%) and activated partial thromboplastin time (APTT, seconds) between patients with BUC and controls.

Table 2 depicts the occurrence of each bleeding symptom and the proportion of manifestations requiring medical intervention at least once.

\section{Parameters of fibrinolysis in patients and controls}

Table 3 shows the results for the fibrinolytic parameters in patients with BUC in comparison to healthy controls. Patients had significantly higher tPA activity than healthy controls. tPA activity was measurable $(>0 \mathrm{U} / \mathrm{mL})$ more frequently in patients than in healthy controls. Levels of tPAPAI-1 complexes were lower in the group of patients with BUC. Levels of fibrinolysis-inhibitors TAFI and $\alpha 2$ antiplasmin were increased in the group of patients and also fibrinogen was higher in patients with BUC in comparison to healthy controls.

In logistic regression analyses, detectable tPA activity $>0 \mathrm{U} /$ $\mathrm{mL}$, low tPA-PAI-1 complexes, increased levels of TAFI, $\alpha 2-$ antiplasmin, and fibrinogen remained significantly associated with BUC also after adjustment for sex (Table 4).

We analyzed the presence of PAI- 1 deficiency defined as plasma levels of PAI-1 antigen $<1 \mathrm{U} / \mathrm{mL}$, a cut-off level also used by Agren et al. [9, 10], as possible cause for BUC. In our population, PAI-1 deficiency occurred with equal frequency in patients and controls (Table 3). Activity of $\alpha 2$-antiplasmin
Table 2 Occurrence of bleeding symptoms and requirement for medical interventions

\begin{tabular}{|c|c|c|c|c|c|}
\hline & \multicolumn{3}{|c|}{ Frequency } & \multicolumn{2}{|c|}{ Medical intervention required } \\
\hline & $N$ & $n$ & $\%$ & $n$ & $\%$ \\
\hline Hematoma/easy bruising & 270 & 176 & 65.2 & 12 & 6.8 \\
\hline Increased postsurgical bleeding $^{\mathrm{a}}$ & 251 & 145 & 57.8 & 79 & 54.5 \\
\hline Abnormal bleeding after tooth extraction ${ }^{a}$ & 239 & 88 & 36.8 & 18 & 20.5 \\
\hline Increased bleeding from small wounds & 270 & 87 & 32.2 & 0 & 0 \\
\hline Epistaxis & 270 & 73 & 27.0 & 25 & 34.2 \\
\hline Increased oral mucosal bleeding & 270 & 32 & 11.9 & 0 & 0 \\
\hline Gastrointestinal bleeding & 270 & 25 & 9.3 & 6 & 24.0 \\
\hline Muscle or joint bleeding & 270 & 4 & 1.5 & 0 & 0 \\
\hline Menorrhagia ${ }^{\mathrm{b}}$ & 227 & 147 & 64.7 & 90 & 61.2 \\
\hline Abnormal postpartum bleeding $\mathrm{g}^{\mathrm{a}, \mathrm{b}}$ & 157 & 53 & 33.8 & 25 & 47.2 \\
\hline
\end{tabular}

${ }^{\text {a }}$ Patients exposed to surgery or tooth extraction or delivery

${ }^{\mathrm{b}}$ All women 
Table 3 Parameters of fibrinolysis in patients with BUC and controls

\begin{tabular}{|c|c|c|c|}
\hline & $\begin{array}{l}\text { Patients } \\
\text { Median ( } 25-75 \text { percentile) }\end{array}$ & $\begin{array}{l}\text { Controls } \\
\text { Median ( } 25-75 \text { percentile) }\end{array}$ & $p$ \\
\hline tPA antigen $(\mathrm{U} / \mathrm{mL})^{\mathrm{a}, *}$ & $1.75(1.10-2.65)$ & $2.26(1.34-3.76)$ & 0.096 \\
\hline tPA activity $(\mathrm{U} / \mathrm{mL})^{\mathrm{a}}$ & $0.00(0.00-0.13)$ & $0.00(0.00-0.00)$ & $<0.001$ \\
\hline PAI-1 antigen $(\mathrm{U} / \mathrm{mL})^{*}$ & $1.9(0.7-5.1)$ & $2.5(0.5-6.5)$ & 0.809 \\
\hline tPA-PAI-1 complex $(\mathrm{ng} / \mathrm{mL})^{\mathrm{a}}$ & $6.86(3.99-10.00)$ & $9.11(7.17-13.12)$ & $<0.001$ \\
\hline TAFI $(\mathrm{ng} / \mathrm{mL})^{\mathrm{b}, *}$ & $18.61(15.80-22.58)$ & $17.03(14.02-20.02)$ & $<0.001$ \\
\hline 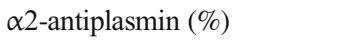 & $102(94-109)$ & $98(90-106)$ & 0.003 \\
\hline Fibrinogen (mg/dL) & $324(281-375)$ & $312(261-343)$ & 0.003 \\
\hline \multirow[t]{2}{*}{ D-dimer $(\mu \mathrm{g} / \mathrm{mL})^{\mathrm{c}, *}$} & $0.28(0.18-0.47)$ & $0.28(0.19-0.40)$ & 0.194 \\
\hline & $n(\%)$ & $n(\%)$ & $p$ \\
\hline PAI-1 antigen $<1 \mathrm{U} / \mathrm{mL}$ & $91(34)$ & $33(34)$ & 0.996 \\
\hline tPA activity $>0 \mathrm{U} / \mathrm{mL}^{\mathrm{a}}$ & $103(48)$ & $23(23)$ & $<0.0001$ \\
\hline
\end{tabular}

$t P A$ tissue plasminogen activator, $P A I-1$ plasminogen activator inhibitor-1, TAFI thrombin activatable fibrinolysis inhibitor

* $\log 2$ transformed variables used for the comparison between the groups

${ }^{\text {a }}$ Available of 213 (78.9\%) patients

${ }^{\mathrm{b}}$ Available of $263(97.4 \%)$ patients

${ }^{\mathrm{c}}$ Available of 262 (97.0\%) patients below normal $(<75 \%)$ was found in five study participants, two patients, and three healthy controls. None of the patients or healthy controls had levels of $\alpha 2$-antiplasmin below $60 \%$.

There was no specific pattern of altered fibrinolysis that would correlate with any of the bleeding symptoms. Patients with skin manifestations had higher levels (median (IQR)) of fibrinogen (330 (294-377) and $307(266-364), p=0.017)$ and D-dimer $(0.32(0.20-0.48)$ and $0.23(0.16-0.35), p=0.003)$ than patients without skin manifestations. Likewise, levels of TAFI (19.86 (16.34-25.77) and 18.42 (15.49-21.88), $p=0.023)$ and D-dimer $(0.36(0.22-0.62)$ and $0.25(0.17-$ $0.41), p<0.001)$ were increased in patients with prolonged bleeding from small wounds compared to those without;

Table 4 Sex-adjusted logistic regression analyses of parameters of fibrinolysis

\begin{tabular}{llll}
\hline & OR & $95 \% \mathrm{CI}$ & $p$ \\
\hline tPA antigen* & 0.87 & $0.72-1.06$ & 0.16 \\
tPA activity $>0 \mathrm{U} / \mathrm{mL}$ & 3.02 & $1.75-5.23$ & $<0.0001$ \\
PAI-1 antigen* & 1.06 & $0.95-1.19$ & 0.30 \\
tPA-PAI-1 complex (ng/mL) & 0.90 & $0.86-0.95$ & $<0.0001$ \\
TAFI* & 2.57 & $1.48-4.46$ & 0.0008 \\
$\alpha 2$-antiplasmin $(\%)$ & 1.03 & $1.01-1.05$ & 0.011 \\
Fibrinogen $(10 \mathrm{mg} / \mathrm{dL})$ & 1.04 & $1.00-1.08$ & 0.030 \\
D-dimer* & 1.05 & $0.83-1.34$ & 0.68 \\
\hline
\end{tabular}

$t P A$ tissue plasminogen activator, $P A I-1$ plasminogen activator inhibitor1, TAFI thrombin activatable fibrinolysis inhibitor

*log2-transformed levels of TAFI were also increased in patients with pathological postsurgical bleeding compared to those with a normal postsurgical course (19.60 (16.34-23.04) and 18.23 (15.20$22.58), p=0.043)$. There was no difference in the assessed fibrinolytic factors between patients with or without increased oral mucosal bleeding, GI bleeding, or prolonged bleeding after tooth extraction. In females, we found no difference in the parameters of fibrinolysis between patients with or without heavy menstrual bleeding. Patients with a history of increased postpartum bleeding had slightly lower levels of D-dimer compared to those with a normal postpartum course $(0.30$ $(0.20-0.47)$ and $0.37(0.25-0.63), p=0.047)$.

\section{Discussion}

We investigated a broad panel of parameters with pro- and anti-fibrinolytic capacity to get a clearer picture about the involvement of fibrinolysis in BUC. The results indicate alterations in the fibrinolytic process as a cause for increased bleeding in patients with BUC. Up to now, parameters involved in the fibrinolytic process were examined only in small patient cohorts with a bleeding tendency, and the results were controversial [10-14, 18].

We found significantly increased tPA activity, lower levels of tPA-PAI-1 complexes, and slightly but significantly increased levels of TAFI, $\alpha 2$-antiplasmin, and fibrinogen in our patients with BUC compared to the group of healthy controls. These factors were also independently associated with BUC in sex-adjusted logistic regression analyses. 
Our findings of increased tPA activity and lower levels of tPAPAI-1 complexes may indicate an impaired inhibition of tPA by PAI-1. This could result from a functional defect of one of the ligands, as there was no difference in PAI-1 activity or PAA antigen levels between patients and controls in our cohort. This impaired inhibition of IPA could result in higher fibrinolytic activity and thus be responsible for the bleeding tendency in our patients with BUC. To the best of our knowledge, we are the first to investigate $\mathrm{PA}$ activity in patients with a bleeding tendency, as previous studies only investigated tPA antigen levels $[10,13,14]$. Likewise, quantitative measurements of tPA-PAI-1 complex levels were only performed in a small number of studies on patients with a bleeding tendency and results are inconclusive $[10,12,18]$. In line with our data, one study reported lower preoperative tPA-PAI-1 complex levels in association with increased bleeding after cardiac surgery [12].

Levels of TAFI and $\alpha 2$-antiplasmin and fibrinogen were slightly, but significantly increased in our patients with BUC compared to controls and remained significantly associated with BUC after adjustment for sex. This increase in anti-fibrinolytic factors is surprising and difficult to explain. Still, it was the most consistent finding when patients with a bleeding tendency were compared to control individuals. In line with our data, increased levels of TAFI and $\alpha 2$-antiplasmin have been reported in women with strong menstrual bleeding [14]. High TAFI levels have also been associated with a more severe bleeding phenotype in patients with hemophilia A and in patients with hereditary mucocutaneous hemorrhages $[10,11]$. In the latter studies, $\alpha 2$ antiplasmin was not investigated. This increased antifibrinolytic capacity might balance the augmented profibrinolytic activity of tPA depicting the steady state in patients with BUC, which prevents spontaneous and severe bleeding episodes. Additional triggers, such as invasive procedures, minimal trauma, or minor injuries, might tip this balance towards bleeding, manifesting as a mild-to-moderate bleeding tendency.

We have previously shown that there was no difference in the overall fibrinolytic capacity, represented by the half lysis time $t_{50}$ in the turbidimetric assessment of plasma clot lysis between a subgroup of our patients with BUC and controls. This finding is in line with the results of Wiewel-Verschueren et al. [14] but in contrast to the findings of Szczepaniak et al. [13], both studies on patients with heavy menstrual bleeding. Overall fibrinolytic capacity is determined after addition of rtPA. This profibrinolytic stimulus might mask subtle, but relevant differences of the fibrinolytic activity. Thus, the clot lysis time measured in plasmabased assays might be more an expression of a clot structure with a higher susceptibility to fibrinolysis than an indicator of the individual endogenous fibrinolytic potential.

Our study has several limitations. First, patients and controls were of comparable age, but there was a female predominance in the patient group. Still, the described differences in fibrinolysis parameters remained statistically significant after adjustment for sex. Second, the fibrinolytic activity follows a circadian variation, with peak levels of PAI-1 and PAA antigen and the lowest IPA activity in the morning [19]. We considered that and restricted the blood sampling time to $8.00-10.00$ for both patients and controls. Further limitations include pre-analytical factors in the determination of parameters of fibrinolysis. TPA activity has been reported as unstable, especially in the presence of high PAI-1 levels [20]. Thus, it has been recommended to determine fibrinolytic variables, especially tPA, in samples of acidified citrated plasma (Stabilyte ${ }^{\mathrm{TM}}$ ) [21-24], which inhibits tPA-PAI-1 complex formation and tPA inactivation [20, 25]. However, tPA-PAI-1 complex levels in citrated plasma have been reported to be only marginally increased [23]. Strengths of our study are the large number of well-characterized patients with BUC, and the systematic investigation of multiple parameters known to regulate fibrinolysis.

In summary, we have measured a comprehensive panel of parameters to assess fibrinolysis in a large cohort of patients with a mild-to-moderate bleeding tendency of unknown cause. We found alterations in fibrinolysis parameters, indicating increased fibrinolytic activity, but also increased protection against fibrinolysis in patients with BUC in comparison to healthy controls. This observation is novel and might depict the resting state of patients at risk for fibrinolytic bleeding after hemostaseological challenges. The hypothesis of increased fibrinolysis in patients with BUC is supported by the clinical efficacy of anti-fibrinolytic agents, such as tranexamic acid, in patients with mild bleeding symptoms [26-28]. We could not confirm prior reports of PAI-1 deficiency [18] as a risk factor for BUC in our cohort. Our data warrant further studies to investigate and characterize the pathophysiological processes underlying fibrinolysis in patients with BUC.

Acknowledgements Open access funding provided by Medical University Vienna. We thank Tanja Altreiter (Department of Medicine I, Clinical Division of Hematology and Hemostaseology, Medical University Vienna) for proof-reading the manuscript. This study was conducted in cooperation with the MedUni Wien Biobank facility. CSL Behring supported the studies by an unrestricted grant and Stago provided the assays for the determination of TAFI, tPA-PAI-1 complexes, and D-dimer. J. Gebhart received financial support through the Bayer Hemophilia Clinical Training Award (http://www.bayer-hemophilia-awards.com).

Author contributions J.G., S.P., H.H., C.M., and I.P. designed the study; J.G., S.K., S.H., and I.P. contributed patients. H.H. and P.Q. processed and stored the samples. J.G., S.K., A.W., H.H., P.Q., and S.P. performed laboratory analyses. E.E. supported data storage. J.G., E.E., A.K., and I.P. collected and interpreted the data. J.G. and A.K. analyzed the data. J.G., A.K., A.W., and I.P. wrote the manuscript. All authors proof-read the manuscript, agreed with the content, and approved its submission.

\section{Compliance with ethical standards}

Ethical approval All procedures performed in the study were in accordance with the ethical standards of the institutional research committee (ethikkommission.meduniwien.ac.at) and with the 1964 Helsinki declaration and its later amendments or comparable ethical standards. Written informed consent was obtained from all individual participants before inclusion in the study. 
Funding CSL Behring supported the studies by an unrestricted grant and Stago provided the assays for the determination of TAFI, tPA-PAI-1 complexes, and D-dimer.

Conflict of interests The authors declare that they have no competing interests.

Open Access This article is distributed under the terms of the Creative Commons Attribution 4.0 International License (http:// creativecommons.org/licenses/by/4.0/), which permits unrestricted use, distribution, and reproduction in any medium, provided you give appropriate credit to the original author(s) and the source, provide a link to the Creative Commons license, and indicate if changes were made.

\section{References}

1. Quiroga T, Mezzano D (2012) Is my patient a bleeder? A diagnostic framework for mild bleeding disorders. Hematology Am Soc Hematol Educ Program 2012:466-474

2. Leebeek FW, Stibbe J, Knot EA, Kluft C, Gomes MJ, Beudeker M (1988) Mild haemostatic problems associated with congenital heterozygous alpha 2-antiplasmin deficiency. Thromb Haemost 59(1):96-100

3. Carpenter SL, Mathew P (2008) Alpha2-antiplasmin and its deficiency: fibrinolysis out of balance. Haemophilia : the official journal of the World Federation of Hemophilia 14(6):1250-1254

4. Fay WP, Shapiro AD, Shih JL, Schleef RR, Ginsburg D (1992) Brief report: complete deficiency of plasminogen-activator inhibitor type 1 due to a frame-shift mutation. N Engl J Med 327(24): 1729-1733

5. Mehta R, Shapiro AD (2008) Plasminogen activator inhibitor type 1 deficiency. Haemophilia : the official journal of the World Federation of Hemophilia 14(6): 1255-1260

6. Schleef RR, Higgins DL, Pillemer E, Levitt LJ (1989) Bleeding diathesis due to decreased functional activity of type 1 plasminogen activator inhibitor. J Clin Invest 83(5):1747-1752

7. Zhang ZY, Wang ZY, Dong NZ, Bai X, Zhang W, Ruan CG (2005) A case of deficiency of plasma plasminogen activator inhibitor-1 related to Ala15Thr mutation in its signal peptide. Blood coagulation \& fibrinolysis : an international journal in haemostasis and thrombosis 16(1):79-84

8. Bauduer F, Menard F, Mimoun A (2015) Plasminogen activator inhibitor type 1 deficiency revealed by severe bleeding after prostatectomy in a 76-year-old male. Blood coagulation \& fibrinolysis : an international journal in haemostasis and thrombosis 26(3):350-351

9. Agren A, Wiman B, Schulman S (2007) Laboratory evidence of hyperfibrinolysis in association with low plasminogen activator inhibitor type 1 activity. Blood Coagul Fibrinolysis 18(7):657-660

10. Grunewald M, Siegemund A, Grunewald A, Konegan A, Koksch M, Griesshammer M (2002) Paradoxical hyperfibrinolysis is associated with a more intensely haemorrhagic phenotype in severe congenital haemophilia. Haemophilia 8(6):768-775

11. Matus V, Willemse J, Quiroga T, Goycoolea M, Aranda E, Panes O, Pereira J, Hendriks D, Mezzano D (2009) Procarboxypeptidase U (TAFI) and the Thr325Ile proCPU polymorphism in patients with hereditary mucocutaneous hemorrhages. Clinica chimica acta; international journal of clinical chemistry 401(1-2):158-161

12. Ozolina A, Strike E, Jaunalksne I, Krumina A, Bjertnaes LJ, Vanags I (2012) PAI-1 and t-PA/PAI-1 complex potential markers of fibrinolytic bleeding after cardiac surgery employing cardiopulmonary bypass. BMC Anesthesiol 12:27

13. Szczepaniak P, Zabczyk M, Undas A (2015) Increased plasma clot permeability and susceptibility to lysis are associated with heavy menstrual bleeding of unknown cause: a case-control study. PLoS One 10(4): $\mathrm{e} 0125069$

14. Wiewel-Verschueren S, Knol HM, Lisman T, Bogchelman DH, Kluin-Nelemans JC, van der Zee AG, Mulder AB, Meijer K (2014) No increased systemic fibrinolysis in women with heavy menstrual bleeding. Journal of thrombosis and haemostasis : JTH 12(9):1488-1493

15. Rodeghiero F, Castaman G, Tosetto A, Batlle J, Baudo F, Cappelletti A, Casana P, De Bosch N, Eikenboom JC, Federici AB, Lethagen S, Linari S, Srivastava A (2005) The discriminant power of bleeding history for the diagnosis of type 1 von Willebrand disease: an international, multicenter study. Journal of thrombosis and haemostasis : JTH 3(12):2619-2626

16. Ay C, Haselbock J, Laczkovics C, Koder S, Pabinger I (2011) Thrombin generation in patients with a bleeding tendency of unknown origin. Ann Hematol 90(9):1099-1104

17. Gebhart J, Laczkovics C, Posch F, Ay C, Reitter-Pfoertner SE, Haslacher H, Muszbek L, Wolberg AS, Pabinger I (2015) Plasma clot properties in patients with a mild-to-moderate bleeding tendency of unknown cause. Ann Hematol 94(8):1301-1310

18. Agren A, Wiman B, Stiller V, Lindmarker P, Sten-Linder M, Carlsson A, Holmstrom M, Odeberg J, Schulman S (2006) Evaluation of low PAI-1 activity as a risk factor for hemorrhagic diathesis. Journal of thrombosis and haemostasis : JTH 4(1):201-208

19. Angleton P, Chandler WL, Schmer G (1989) Diurnal variation of tissue-type plasminogen activator and its rapid inhibitor (PAI-1). Circulation 79(1):101-106

20. Chandler WL, Trimble SL, Loo SC, Mornin D (1990) Effect of PAI-1 levels on the molar concentrations of active tissue plasminogen activator (t-PA) and t-PA/PAI-1 complex in plasma. Blood 76(5):930-937

21. Chandler WL, Loo SC, Nguyen SV, Schmer G, Stratton JR (1989) Standardization of methods for measuring plasminogen activator inhibitor activity in human plasma. Clin Chem 35(5):787-793

22. Chandler WL, Schmer G, Stratton JR (1989) Optimum conditions for the stabilization and measurement of tissue plasminogen activator activity in human plasma. J Lab Clin Med 113(3):362-371

23. Nilsson TK, Boman K, Jansson JH, Thogersen AM, Berggren M, Broberg A, Granlund A (2005) Comparison of soluble thrombomodulin, von Willebrand factor, tPA/PAI-1 complex, and high-sensitivity CRP concentrations in serum, EDTA plasma, citrated plasma, and acidified citrated plasma (Stabilyte) stored at -70 degrees C for 8-11 years. Thromb Res 116(3):249-254

24. Nilsson TK, Mellbring G (1989) Impact of immediate acidification of blood on measurement of plasma tissue plasminogen activator (tPA) activity in surgical patients. Clin Chem 35(9):1999

25. Ranby M, Sundell IB, Nilsson TK (1989) Blood collection in strong acidic citrate anticoagulant used in a study of dietary influence on basal tPA activity. Thromb Haemost 62(3):917-922

26. Rydz N, James PD (2012) Approach to the diagnosis and management of common bleeding disorders. Semin Thromb Hemost 38(7):711-719

27. Leminen H, Hurskainen R (2012) Tranexamic acid for the treatment of heavy menstrual bleeding: efficacy and safety. International journal of women's health 4:413-421

28. Zahed R, Moharamzadeh P, Alizadeharasi S, Ghasemi A, Saeedi M (2013) A new and rapid method for epistaxis treatment using injectable form of tranexamic acid topically: a randomized controlled trial. Am J Emerg Med 31(9):1389-1392 